\title{
Commentaries
}

\section{The failing malignant liver}

Acute liver failure, defined as hepatic encephalopathy developing within 12 weeks of jaundice in the absence of a previous history of symptomatic liver disease, ${ }^{1}$ is a medical emergency. Accurate recognition of the underlying disease is crucial to enable specific therapy, when available, to be instituted. Rowbotham et al (see page 576), in the most comprehensive examination of this topic to date, discuss the importance of malignant hepatic infiltration as a cause of acute liver failure. This is, however, an undoubtedly rare cause for this presentation in the UK, responsible for only $0.44 \%$ of all cases of liver failure admitted to the King's Unit since 1978.

This syndrome can occur in a number of scenarios. Firstly and most importantly, patients with no previous history of malignancy may develop fulminant liver failure and present as a diagnostic problem. Secondly, acute liver failure may develop in a patient with previously diagnosed malignancy but without known liver involvement. Finally, fulminant liver failure may be seen as a terminal event in a patient already known to have primary or secondary hepatic malignancy. Such patients, although not fulfilling the strict diagnostic criteria for acute liver failure, may provide further insight into the likely mechanisms of liver failure in malignancy.

In a patient presenting with acute liver failure with no cause apparent, the diagnosis of hepatic infiltration should be considered but may be surprisingly difficult to make. Certain clinical and biochemical parameters may heighten the suspicion of infiltrative disease. All patients in the King's series had a non-specific prodromal illness for 1-8 weeks prior to presentation. Jaundice tends to be milder compared with other causes of acute liver failure whereas hepatomegaly is more pronounced (indeed in many cases of acute liver failure the liver is small). In contrast to liver failure resulting from paracetemol overdose, signs of cerebral oedema are rare in malignancy with only one case report ${ }^{2}$ describing the occurrence of pupillary abnormalities and decerebrate posturing as a terminal event in a patient with non-Hodgkin's lymphoma. The finding of lymphadenopathy and splenomegaly clinically or on imaging is helpful in recognising cases owing to lymphoma, but is not helpful in the diagnosis of other malignancies. Abnormal liver texture was noted on imaging in less than half the cases described by Rowbotham et al. Massive increases in aminotransferases (up to 100-fold elevated) can occur with malignant infiltration ${ }^{34}$ and would help to exclude acute alcoholic hepatitis, which may occasionally be misdiagnosed in this setting. ${ }^{6}$ Lactic acidosis and a disproportionately raised lactate dehydrogenase are suggestive of haematological malignancy. ${ }^{7}$ Sugano and Suzuki recently reported the finding of a notable increase of the brain isozyme of creatine kinase (CK-BB) in a patient with hepatic failure and non-Hodgkin's lymphoma and suggest it may be useful as a marker of ischaemia of hepatic sinusoidal cells. ${ }^{8}$ The specificity of this test needs to be evaluated. No clinical or biochemical features are sufficiently specific to obviate the importance of a tissue diagnosis. Reassuringly, in the King's series, there were no complications reported from liver biopsy (percutaneous, transjugular or via laparotomy) in the eight patients in whom it was performed. Frequently there may be only a narrow window of opportunity for safe biopsy in these rapidly deteriorating patients. ${ }^{3}$

Alternate sources of tissue for diagnostic purposes include bone marrow and lymph nodes.

In the second group of patients a history of previous malignancy will alert the clinician to the possible infiltrative cause of liver failure. Haematological malignancies are implicated most commonly, in particular Hodgkin's and non-Hodgkin's lymphoma, as well as malignant histiocytosis, acute leukaemia and, recently described, Richter's transformation of chronic lymphocytic leukaemia. ${ }^{3}$

Other less common causes are breast, colon, gastric, and small cell carcinoma. ${ }^{4}$ Occasionally, the history of malignancy may be remote and the patient believed to be tumour free. ${ }^{10}$ Alternately, the patient may have a predisposing condition to malignancy only, such as coeliac disease $^{2}$ or autoimmune haemolytic anaemia (Rowbotham et al). The differential diagnosis of acute liver failure in patients with malignancy should also include the possibility of chemotherapy or radiotherapy induced liver failure, or of reactivation of underlying chronic hepatitis $\mathrm{B}$ liver disease, described as a complication of chemotherapy. ${ }^{11}$

The third group of patients is those where hepatic failure occurs as a terminal event in the setting of advanced malignant disease. This may be under-recognised as a clinical entity for in such patients hepatic coma may be mistakenly attributed to severe constitutional disease, cerebral metastases, drugs or sepsis. ${ }^{9}$ Eras and Sherlock found in a retrospective study that $7.2 \%$ of hospitalised patients with hepatic metastases had evidence, albeit only clinical, of hepatic encephalopathy but in over half this was not recognised or treated. ${ }^{9}$

A number of mechanisms are proposed to explain the pathogenesis of infiltrative liver failure and these are clearly discussed by Rowbotham et al. Massive cytokine release has been implicated as a cause of liver failure and this theory is particularly attractive given the fact that responsible cytokines, such as interleukin 2, are released by lymphomas, the commonest malignancy responsible for acute liver failure. This mechanism explains why liver failure can occur despite a relatively low, or even absent tumour burden in the liver. ${ }^{12}$ Cytokine release can cause liver failure by damaging bile ducts both directly and via recruitment of effector cells, and by activation of leucocytes and hepatic sinusoidal cells, thus impeding hepatic sinusoidal microcirculation. Ultimately, liver failure occurs as a result of ischaemia, probably as a consequence both of cytokine release and on occasion from direct physical compression of hepatic sinusoids by the expanding tumour mass. ${ }^{10}$ Acute liver failure can occasionally reverse very quickly with appropriate chemotherapy, ${ }^{3}$ consistent with the mechanisms outlined above, whereas if liver failure occurred as a result of the loss of a critical mass of hepatocytes due to replacement with malignant cells, rapid improvement would not be anticipated. The direct effect of tumour infiltration with replacement of hepatocytes is probably more important as a mechanism in nonhaematological malignancies such as small cell carcinoma. ${ }^{413}$ Liver failure may also occur due to ischaemia produced by tumour emboli compromising the portal 
venous circulation, particularly in primary hepatocellular carcinoma. ${ }^{14} \mathrm{~A}$ further mechanism proposed is liver failure due to hepatic venous obstruction (secondary to emboli, hypercoagulability or a compressive mass).

Unfortunately, the prognosis of patients with acute liver failure resulting from malignant infiltration is dismal. The few survivors are those with haematological malignancies ${ }^{3} 7$ in whom, crucially, the diagnosis was made promptly and treatment instituted. The only survivor in the King's series (Rowbotham et al) was a patient where chemotherapy was initiated by the referring hospital prior to transfer. Unfortunately, survival is not reported for patients with non-haematological malignancies, such as in our own personal experience with two patients with small cell and breast carcinoma. Despite being unable to offer potentially curative treatment in this group, recognition of the diagnosis is important to ensure that these patients are not inappropriately listed for liver transplantation.

B C SMITH O F W JAMES

Centre for Liver Research,

The Medical School,

University of Newcastle upon Tyne,

Newcastle upon Tyne NE2 4HH, UK
1 O'Grady J, Schalm S, Williams R. Acute liver failure: redefining the syndromes. Lancet 1993;342:273-5.

2 Braude S, Portmann B, Gimson A, et al. Fulminant hepatic failure in nonHodgkin's lymphoma. Postgrad Med f 1982;58:301-4.

3 Shehab T, Kaminski M, Lok A. Acute liver failure due to hepatic involvement by hematologic malignancy. Dig Dis Sci 1997;42:1400-5.

4 Krauss E, Ludwig P, Sumner H. Metastatic carcinoma presenting as fulminant hepatic failure. Am f Gastroenterol 1979;72:651-4.

5 Pal N, Whittingham G, Paley R. Hepatic coma due to metastases. BMF 1965;i:168.

6 Colby T, LaBrecque D. Lymphoreticular malignancy presenting as fulminant hepatic disease. Gastroenterology 1982;82:339-45.

7 Ghosh P, Fox I, Rader A, et al. Fulminant hepatic failure as the initial manifestation of non-Hodgkin's lymphoma. Am $\mathcal{F}$ Gastroenterol 1997;90: 2207-9.

8 Sugano S, Suzuki T. Creatine kinase-BB: Markedly increased in fulminant hepatic failure caused by massively disseminated lymphoma cells in the liver. Hepatology 1995;22:679-80.

9 Eras P, Sherlock P. Hepatic coma secondary to metastatic liver disease. Ann Intern Med 1971;74:581-3.

10 Woolf G, Petrovic L, Rojter S, et al. Acute liver failure due to lymphoma. Dig Dis Sci 1994;39:1351-8.

11 Soh L, Ang P, Sng I et al. Fulminant hepatic failure in non-Hodgkin's lymphoma patients treated with chemotherapy. Eur f Cancer 1992;28A: 1338-9.

12 Fang J, Lau J, Lai C. Fulminant hepatic failure in nonmetastatic renal cell carcinoma. Dig Dis Sci 1992;37:474-7.

13 Harrison $\mathrm{H}$, Middleton H, Crosby J, et al. Fulminant hepatic failure: an unusual presentation of metastatic liver disease. Gastroenterology 1981;80: 820-5.

14 Okuda $\mathrm{K}$, Musha $\mathrm{H}$, Kanno $\mathrm{H}$, et al. Localized submassive liver cell necrosis as a terminal event of liver cancer. Cancer 1966;37:1965-72.

\section{Gut epithelium: food processor for the mucosal immune system?}

To those outside the field of mucosal immunology it must seem incredible that we do not yet understand something so basic as the interaction of protein antigens with the intestinal epithelium. Although we now know in some detail the mechanisms by which the mucosal immune system is regulated and we can construct elegant adjuvantpeptide fusion proteins with systems capable of precise delivery in conjunction with what we think are appropriate cytokines, we know very little of what happens to even the most simple protein antigens during transport. Conversely, the emergence of several good rodent models of intestinal inflammation has had a major impact on our understanding of inflammatory bowel disease pathogenesis, but without further information on $\mathrm{T}$ cell-epithelial cell-antigen interactions, the vital mechanisms of the initial "barrier" defect will continue to elude us. Are antigens selectively degraded during transport across the epithelium? If so, is this essential to the maintenance of immune homeostasis? How is the relative transport of intact protein, peptides and amino acids controlled and is the maintenance of a "normal" flora important in this control? All these are vital questions to which we have few sensible responses.

Macromolecular transport by the adult gut epithelium was established in the 1970 s, in particular by the extensive work from Allan Walker's laboratory. ${ }^{1}$ Some answers (but even more questions!) have been provided in the past decade by workers analysing antigen processing and presentation by isolated gut epithelial cells. ${ }^{2}$ These studies, which were stimulated by the discovery of MHC antigen expression by absorptive enterocytes, give some indication that antigens are modified by enterocytes, but prolonged analysis of isolated enterocytes in primary culture is impossible. Studies in vivo, with induction of mucosal tolerance as a readout, have shown that tolerance after feeding is dependent on gut processing of fed antigen at some level and indeed that gut processed antigen which has been absorbed into the serum has distinct characteristics and can transfer tolerance, ${ }^{34}$ but the role of the epithelium in this processing has not been investigated further. The reasons are complex, but largely boil down to a lack of appropriate in vitro systems for analysing such a complex tissue. The development of polarised intestinal epithelial cell lines has supplied potential models, but the questions asked must be appropriate to the model and the predictions made must be cautious.

In this issue (see page 538), Terpend et al have used HT-29 epithelial cell monolayers in Ussing chambers to determine the transport of horseradish peroxidase (HRP) and its products across a model intestinal epithelium. They show that under control conditions, HRP is transported from apical to basolateral surfaces as intact molecule, peptides and amino acids. The peptide products have been characterised by molecular weight using steric exclusion HPLC as a peak containing a heterogenous group of degradation products and a separate peak comprising peptides of around eight amino acids. This latter group represents peptides capable of binding to MHC molecules for presentation to $\mathrm{T}$ cells.

Based on these results, we can speculate that if HT-29 in vitro mimics the transport characteristics of small bowel epithelium in vivo, then the epithelium is capable of taking an active part in processing dietary proteins to immunologically relevant peptides. Other work from this same group suggests that the balance which the epithelium maintains between absorbed native protein and absorbed degradation products may be important in preventing food allergy. ${ }^{5}$ However, the "if" is considerable in a tissue in which the maintenance of cellular differentiation, polarity, microvillus development, ion channel integrity, tight junction complexity, basement membrane interaction, and cytokine profile (to name but a few known parameters) are essential to stable function. The authors have gone to considerable lengths to control for the limitations of the system and to analyse peptides, monolayer 
integrity and proteolytic enzymes using appropriate methodology - unfortunately, not universal practice among the increasing numbers of workers using epithelial cell lines, often inappropriately, for analysis of gut epithelial function. We can only make the leap from such relatively simple systems to confident statements on the enormously complex in vivo situation if such appropriate methods and assumptions are used.

The results also show, not surprisingly, that basolateral interferon $\gamma($ IFN- $\gamma)$ causes the monolayer to become leaky and, interestingly, that this induced leakiness does not affect the relative proportions of transported intact HRP (presumed paracellularly) and degraded HRP (presumed transcellularly). Recent work by other groups has shown that transcellular and paracellular HRP transport and the relation of native to degraded antigen are differentially modulated in rat intestine after mast cell degranulation, ${ }^{6}$ or in biopsy tissue from individuals with atopic eczema. ${ }^{7}$ Clearly, the effects of different types of local immune activation on the degradation of antigen by enterocytes and on tight junction effectiveness are complex.

The increased transport of HRP with IFN- $\gamma$ treatment in Terpend et al's study was not reflected by changes in the intracellular proteases analysed. More detailed analysis of these enzymes and changes in their expression due to proinflammatory cytokines is both suggested by these results and essential to understand the epithelial degradative capacity. The lack of effect of IFN- $\gamma$ on cathepsin expression in HT-29 confirms the recent report of Hershberg et $a l,{ }^{8}$ but the Hershberg group showed different effects with different cell lines-cathepsin B was increased in T84 cells after IFN- $\gamma$ treatment, but not cathepsins $\mathrm{L}$ or $\mathrm{H}$ or aspartic proteases. Again, this suggests that we must use caution in the wider interpretation of studies using these model systems.

HLA-DR was only present after IFN- $\gamma$ induction in the HT-29 cells. This might indicate that as the proportions of native to degraded protein are similar in control and IFN- $\gamma$ treated cells, MHC class II proteins are not important in providing a protected transport route for certain peptides. ${ }^{9}$ However, the role of MHC class II in enterocytes - it is expressed constitutively in normal human and rodent intestinal epithelium - is a complex issue and will only be resolved by analysis of dietary peptides bound to epithelial MHC class II proteins, as the authors suggest.

This work adds significantly to our understanding of the transport and processing capacities of the intestinal epithelium. Together with previous work with ex vivo epithelial cells and in vivo analyses, it allows us to predict that the gut epithelium is an active participant in the generation and regulation of immune responses to enteric antigens and that these responses can, in turn, feed back and modulate epithelial cell function.

Division of Molecular and Cellular Biology,

Department of Clinical Veterinary Science,

University of Bristol, Langford House,

Langford, Bristol BS40 5DU, UK

1 Walker WA. Role of the mucosal barrier in antigen handling by the gut. In: Brostoff J, Challacombe SJ, eds. Food allergy and intolerance. Eastbourne: Bailliere Tindall, 1987:209-22.

2 Bland PW, Whiting CV. Antigen processing by isolated rat intestinal villus enterocytes. Immunology 1989;68:497-502.

3 Bruce MG, Ferguson A. The influence of intestinal processing on the immunogenicity and molecular size of absorbed, circulating ovalbumin in mice. Immunology 1986;59:295-300.

4 Furrie E, Turner MW, Strobel S. Partial characterisation of a circulating tolerogenic moiety which, after a feed of ovalbumin, suppresses delayed-type hypersensitivity in recipient mice. Immunology $1995 ; 86: 480-6$.

5 Benlounes N, Dupont C, Candahl C, et al. The threshold for immune cell reactivity to milk antigens decreases in cows milk allergy with intestinal reactivity to milk antigens decreases in cows milk
symptoms. F Allergy Clin Immunol 1996;98:781-9.

6 Berin MC, Kiliaan AJ, Yang PC, et al. Rapid transepithelial antigen transport in rat jejunum: Impact of sensitization and the hypersensitivity transport in rat jejunum: Impact of sensitization. Gastroenterology 1997;113:856-64.

7 Majamaa $\mathrm{H}$, Isolauri E. Evaluation of the gut mucosal barrier-evidence for increased antigen transfer in children with atopic eczema. $\mathcal{F}$ Allergy Clin Immunol 1996;97:985-90.

8 Hershberg RM, Framson PE, Cho DH, et al. Intestinal epithelial cells use two distinct pathways for HLA class II antigen processing. 7 Clin Invest 1997;100:204-15.

9 Bland PW. MHC class II expression by the gut epithelium. Immunol Today 1988;9:174-8.

\section{Is the hepatocyte a Trojan horse for hepatitis $\mathrm{C}$ virus?}

Hepatitis $\mathrm{C}$ virus (HCV) is a major public health problem around the world, with a prevalence ranging from 0.5 to $2 \%$ in most developed countries. After acute infection, spontaneous remission is a rare event. Although long term consequences of HCV infection are controversial, there is a consensus emerging that $\mathrm{HCV}$ infection is a progressive disease resulting in liver failure in some but probably in a minority of infected individuals. Patients who lack biochemical evidence of liver injury may be an important subset with a "more benign" natural history.

In the rare situation where spontaneous cure occurs after acute infection, aminotransferase activities normalise and HCV RNA becomes undetectable in serum. By contrast, the serological profile shows minor modifications with time. With chronicity, serum HCV RNA remains detectable in the vast majority of individuals. Undetectable HCV RNA in serum in patients with abnormal liver enzymes may be related to low viraemia, be under the limit of detec- tion of virological assays, or be due to a fluctuating course. In anti-HCV positive individuals with normal enzymes, however, the lack of serum HCV RNA has been interpreted as complete cure of the infection. However, the study by Haydon et al in this issue (see page 570) challenges this interpretation. These investigators have documented HCV RNA in liver in the majority of patients with exposure to HCV (confirmed by RIBA 3) whether or not virus was detectable in serum, suggesting that residual $\mathrm{HCV}$ infection occurs not only among those individuals with abnormal liver enzymes but also among those considered to have "resolved infection".

Although a high correlation exists between third generation assays and HCV RNA in serum, ${ }^{2}$ a small subgroup of patients remains, focused on in this study, which are RIBA 3 positive but HCV RNA negative in serum, regardless of liver enzymes. The question arises whether this represents a past cured infection, mainly in those with normal enzymes, or whether it represents an actual infection with undetectable serum HCV RNA. The authors sought to resolve this question by assessing intrahepatic HCV RNA levels in two different groups of subjects: (i) 12 patients with the above mentioned characteristics, seven of whom had normal aminotransferase activities; and (ii) 86 patients 
with the classic picture of chronic HCV infection (RIBA 3 positive and detectable serum HCV RNA by reverse transcription polymerase chain reaction (RT-PCR)). The main findings can be summarised as follows: (i) $87.5 \%$ of patients without detectable HCV RNA in serum were HCV RNA positive in liver, suggesting that these patients had a true infection; (ii) serum RT-PCR negative and positive groups were similar with regard to demographic, histological and virological data, suggesting that RT-PCR negative patients may indeed have clinically significant liver disease; and (iii) intrahepatic viral levels were not associated with any host or viral factor, including age, sex, duration of disease, mode of $\mathrm{HCV}$ acquisition, alcohol consumption, disease severity, and viral genotype.

This study represents a further step in the understanding of the mechanisms of viral induced liver disease and supports the hypothesis that it is immune mediated rather than cytopathic. ${ }^{3}$ Previous studies done in "apparently healthy blood donors" have reported similar results with high rates of liver damage in anti-HCV positive individuals with or without viraemia and normal aminotransferase activities. ${ }^{1}$ When analysing these data, however, several factors should be clearly defined in order to compare the results. The first is the definition of persistently normal aminotransferase activities. Due to the fluctuating nature of chronic $\mathrm{HCV}$ infection, repeat tests for alanine aminotransferase (ALT) activities are needed in order to differentiate those individuals with intermittently raised ALT from those who have truly persistently normal activities. The second issue is RT-PCR variability. Undetectability of serum HCV RNA by RT-PCR may be a false negative result from inadequate specimen handling and storage, type of sample used, incorrect design of amplification primers or serum contamination with interfering substances. ${ }^{4}$ Indeed, sensitivity of RT-PCR assays has been shown to vary from one centre to another, especially when in-house methods are applied. ${ }^{5}$ Furthermore, although PCR is a highly sensitive technique, there is a limit of detection below which a very low rate of replication may not be detected. Finally, absence of viraemia may result from time dependent fluctuations in serum HCV RNA levels. The methods used by Haydon et al were meticulously applied state of the art techniques, and HCV RNA was assessed at three different time points in order to eliminate the bias of time dependent variations in viraemia. Moreover, intrahepatic HCV RNA was detected in the majority of patients with histologically confirmed liver damage, suggesting that HCV RNA was not detected in serum as a result of a low viraemic status rather than methodological difficulties.

Despite improvements in the diagnosis of $\mathrm{HCV}$ infection, we have little information about factors which determine progression of disease. ${ }^{3}$ An intriguing question which is under investigation is the relation between genotype, HCV replication and histological severity. Conflicting results exist as to the association between genotype 1 (subtype 1b) infection and severe liver disease, independent of viral load. In some of the studies where an association has been found, patients with HCV genotype $1 \mathrm{~b}$ had higher levels of viraemia, suggesting that this particular genotype was capable of inducing more severe liver disease through its greater replicative capacity. Indeed, other studies have found an association between genotype 1 and higher HCV RNA levels. Studies relating genotype to HCV RNA levels or assessing the independent effect of both factors must be interpreted with caution however, as some primers and/or probes used to quantify HCV RNA hybridise with different efficiency to different genotypes. ${ }^{6}$ More recent studies, using appropriate correction factors for the bDNA version 1.0 assays or using different means of quantitation, have not found differences in HCV RNA levels among genotypes. ${ }^{7}$ Haydon et al, using limiting dilution PCR with primers from 5 'NCR provide further support for this observation.

The association between HCV RNA levels and disease severity, independent of genotype, is also controversial. One possible reason is the existence of inaccuracies in viral quantitation. In order to avoid them meticulous attention must be given to processing of serum samples, ${ }^{3}$ yet methods of processing have varied from study to study. An inherent assumption in all the studies is that HCV RNA quantified in serum reflects HCV RNA in liver, and that HCV RNA detected in a needle biopsy sample is representative of HCV RNA levels in the liver as a whole, both of which have been shown in recent studies. ${ }^{8}$ Haydon et al support this assumption as serum RT-PCR positive patients had higher levels of intrahepatic HCV RNA than serum negative ones.

Thus it seems that quantitation of HCV RNA in serum is a reasonable reflection of viral quantitation in hepatocytes with non-viraemic anti-HCV positive patients simply representing the lower end of the spectrum of patients with $\mathrm{HCV}$ infection - that is, those with very low replication rate below the limit of detection of the PCR. A comparable group of patients are those who respond biochemically and virologically to interferon. Indeed, relapse has been shown to occur, even several years after patients seem to have achieved a treatment induced "cure", emphasising the concept that negative serum HCV RNA does not necessarily imply complete cure. ${ }^{9}$ In some of these cases, liver HCV RNA is still present, suggesting that the rate of replication is too low to be detected by current assays. In contrast, negative HCV RNA both in liver and serum has been associated with sustained response to treatment. ${ }^{10}$

In conclusion, (i) the detection of HCV RNA in liver demonstrates residual $\mathrm{HCV}$ infection in patients who have up until now be considered to have resolved infection. These data explain the presence of liver injury in the vast majority of so called "healthy HCV carriers", and raises serious questions as to whether a true "HCV healthy carrier state" really exists. Only careful prospective natural history studies of patients with confirmed seropositivity for HCV yet persistently normal liver enzymes or undetectable serum HCV RNA will be able to answer these questions. (ii) The virological, epidemiological and histological similarities between viraemic and non-viraemic anti-HCV individuals and the absence of a correlation between intrahepatic HCV RNA levels and disease severity represent additional data that favour an immune mediated mechanism of liver injury in $\mathrm{HCV}$ infection. (iii) Antiviral therapy should be considered for patients with HCV related liver disease whether or not they have delectable virus in serum as the majority will have virus in liver and histological evidence of hepatic disease. (iv) Similar precautions and advice regarding transmission should be applied to anti-HCV (RIBA) positive patients whether or not virus can be detected in serum.

M BERENGUER T L WRIGHT

111B, GI Unit,

Veterans Administration Medical Center,

4150 Clement Street,

San Francisco, CA 94121, USA

1 Prieto M, Olaso V, Verdú C, et al. Does the healthy hepatitis C virus carrier state really exist? An analysis using polymerase chain reaction. Hepatology 1995;22:413-17.

2 Younossi Z, McHutchison J. Serological tests for HCV infection. Viral Hepatitis Reviews 1996;2:161-73.

3 González-Peralta RP, Lau JYN. Pathogenesis of hepatocellular damage in chronic hepatitis C virus infection. Seminars in Gastrointestinal Disease 1995;6:28-34. 
4 Pawlotsky JM. Measuring hepatitis C viremia in clinical samples: can we trust the assays. Hepatology 1997;26:1-4.

5 Zaaijer HL, Cuypers HTM, Reesink HW, et al. Reliability of polymerase chain reaction for defection of hepatitis C virus. Lancet 1993;341:722-4. 6 Ohno T, Lau JYN. The "gold standard" accuracy, and the current concepts: hepatitis C virus genotype and viremia. Hepatology 1996;24:1312-15

7 Hawkins A, Davidson F, Simmonds P. Comparison of plasma virus loads among individuals infected with hepatitis $\mathrm{C}$ virus (HCV) genotypes 1,2 , and 3 by quantiplex HCV RNA assays versions 1 and 2, Roche monitor assay, and an in-house limiting dilution method. $\mathcal{F}$ Clin Microbiol 1997;35: $187-92$
8 Terrault N, Dailey P, Ferrell L, et al. Quantification and intrahepatic distribution of HCV RNA in whole livers. $\mathcal{7}$ Med Virol 1997;51:217-24.

9 Lau JYN, Mizokami M, Ohno T, et al. Discrepancy between biochemical and virological responses to interferon alfa in chronic hepatitis C. Lancet 1993;342:1208-9.

10 Marcellin P, Boyer N, Gervais A, et al. Long-term histologic improvement and loss of detectable intrahepatic HCV RNA in patients with chronic hepatitis $\mathrm{C}$ and sustained response to interferon alpha therapy. Ann Intern Med 1997;127:875-81.

\section{Pathogenesis of inflammatory bowel disease: transcription factors in the spotlight}

Dysregulated cytokine production by mucosal lymphocytes and macrophages has been implicated in the pathogenesis of both Crohn's disease and ulcerative colitis, the two major forms of human inflammatory bowel disease (IBD). ${ }^{1}$ Over the past few years, various murine models of chronic intestinal inflammation resembling IBD have been discovered which have provided important clues as to the nature of this dysregulation and to its possible treatment with cytokines. ${ }^{2}$ Thus, in studies of several of the models most closely resembling Crohn's disease it has been shown that production of large amounts of Th1-type cytokinesfor example, interferon $\gamma$, by T cells is a major and essential feature of the inflammation. ${ }^{2}$ In addition, it has been shown that this disease causing Th1 cytokine response can be counteracted by induction of a suppressor response involving the generation of $\mathrm{T}$ cells producing Th2-type cytokines or transforming growth factor $\beta .^{3}$ Finally, it has been shown that Th1 cytokine production in these models is triggered by increased production of interleukin (IL) 12, a cytokine that plays a major role in driving $\mathrm{T}$ cell differentiation. ${ }^{3}$ Taken together, these data may permit the rational design of cytokine based treatment strategies for patients with IBD, and clinical trials with recombinant IL- $10^{4}$ and antibodies to tumour necrosis factor have already been undertaken. ${ }^{56}$

The above data predict abnormal regulation of cytokine gene transcription in patients with IBD. Thus, the focus of recent research has been on the identification of signalling pathways and transcription factors that govern cytokine gene transcription in IBD. Schreiber et al, in this issue (see page 477), report increased expression of the p65 subunit of the transcription factor $\mathrm{NF}-\kappa \mathrm{B}$ in intestinal biopsy specimens from patients with active Crohn's disease. These results support and extend previous data indicating that concentrations of the $\mathrm{NF}-\kappa \mathrm{B}$ p65 subunit are strikingly increased in lamina propria macrophages of patients with this disease. ${ }^{7}$ Furthermore, these findings are consistent with immunohistochemical data indicating increased expression of the NF- $\kappa \mathrm{B}$ p65 subunit in Crohn's disease. ${ }^{8}$

$\mathrm{NF}-\kappa \mathrm{B}$ is a pleiotropic transcriptional activator of various gene promoters for adhesion molecules and cytokines that can be induced by both cytokines and bacterial polymers. ${ }^{9}{ }^{10}$ Many NF- $\kappa B$ family members are able to form homo- or heterodimers with different binding specificity toward variants of a characteristic decameric DNA recognition site (consensus sequence: GGGRNNTYCC). A wide range of stimuli promotes $\mathrm{NF}-\kappa \mathrm{B}$ nuclear translo- cation by a mechanism that involves the ubiquitinproteosome pathway and I $\mathrm{B}$ phosphorylation which seems to target $\mathrm{I} \kappa \mathrm{B}$ for degradation and leads to dissociation of $I \kappa B$ from the $N F-\kappa B$ complex and subsequent translocation of $\mathrm{NF}-\kappa \mathrm{B}$ to the nucleus. Given the functional importance of $\mathrm{NF}-\kappa \mathrm{B}$ in controlling transcriptional activity of promoters of various proinflammatory cytokines, the above data suggest that NF- $\kappa B$ plays a key role in the pathogenesis of chronic intestinal inflammation.

Inhibition of NF- $\mathrm{NB}$ activity has been recently suggested to be a major component of the anti-inflammatory activity of glucocorticoids, which are frequently used for treatment of chronic intestinal inflammation in humans. ${ }^{19}$ These data are consistent with Schreiber et al's results showing downregulation of NF- $\mathrm{NB}$ DNA binding activity on treatment of intestinal cells with corticosteroids. Although activation of the $\mathrm{NF}-\kappa \mathrm{B}$ p65 subunit is not specific to patients with IBD, its perpetuated activation makes it an attractive target for therapeutic intervention. Thus, downregulation of NF- $\mathrm{NB}$ binding activity emerges as a key event in the control of chronic intestinal inflammation in humans and potential strategies to inhibit $\mathrm{NF}-\kappa \mathrm{B}$ activity more specifically are desirable. Such strategies include antioxidants proteosome inhibitors, inhibition of $\mathrm{NF}-\kappa \mathrm{B}$ by I $\kappa \mathrm{B}$ expression vectors, and antisense DNA targeting of NF- $\mathrm{NB}$ p65. ${ }^{71011}$ Thus, the above data suggest that targeting of $\mathrm{NF}-\kappa \mathrm{B}$ may be a novel molecular approach for the treatment of patients with IBD.

It is clear from various new studies that the p65 subunit of $N F-\kappa B$ is not the only transcription factor involved in the pathogenesis of IBD and various other regulatory proteins (for example, AP-1, NFAT and STAT proteins) are very likely to play a key role as well. It seems that we will gain new, fundamental insights into the pathogenesis, prognosis and treatment of IBD by analysing further transcriptional regulatory mechanisms in chronic intestinal inflammation. This may allow us to understand the basis for altered cytokine gene transcription in patients with IBD. Furthermore, such studies will hopefully permit the design of new treatment strategies that have added specificity but reduced toxicity compared with standard immunosuppressive therapy.

Laboratory of Immunology,

M F NEURATH

I. Medical Clinic,

University of Mainz,

Langenbeckstrasse,

55101 Mainz, Germany

1 Strober W, Neurath MF. Immunological diseases of the gastrointestinal tract. In: Rich RR, ed. Clinical immunology. St Louis: Mosby, 1995:140128

2 Elson CO, Sartor RB, Tennyson GS, et al. Experimentel models of inflammatory bowel disease. Gastroenterology 1995;109:1344-67.

3 Strober W, Kelsall BL, Fuss I, et al. Reciprocal IFN- $\gamma$ and TGF- $\beta$ responses regulate the occurrence of mucosal inflammation. Immunol Today 1997;18: $61-4$. 
4 van Deventer SJH, Elson CO, Fedorak RN. Multiple doses of intravenous interleukin-10 in steroid-refractory Crohn's disease. Gastroenterology 1997; 113:383-9.

5 van Dullemen HM, van Deventer SJH, Hommes DW, et al. Treatment of Crohn's disease with anti-tumor necrosis factor chimeric monoclonal antibody. Gastroenterology 1995;109:129-35.

6 Targan SR, Hanauer SB, van Deventer SJH, et al. A short-term study of chimeric monoclonal antibody cA2 to tumor necrosis factor alpha for Crohn's disease. N Engl f Med 1997;337:1029-35.

7 Neurath MF, Pettersson S, Meyer zum Büschenfelde KH, et al. Local administration of antisense phosphorothioate oligonucleotides to the p65 subunit of NF-кB abrogates experimental colitis in mice. Nat Med 1996;2: 998-1004

8 Neurath MF, Fuss I, Schürmann G, et al. Cytokine gene transcription by NF- $\mathrm{B}$ family members in patients with inflammatory bowel disease. Ann NY Acad Sci (in press)

9 Baeuerle PA, Henkel T. Function and activation of NF- $\kappa B$ in the immune system. Ann Rev Immunol 1994;12:141-79.

10 Jobin C, Haskill S, Mayer L, et al. Evidence for altered regulation of IאB $\alpha$ degradation in human colonic epithelial cells. F Immunol 1997;158:226-34.

11 Jobin C, Hellerbrand C, Licato LL, et al. NF-אB mediates cytokine induced expression of ICAM-1 in an intestinal epithelial cell line, a process blocked by proteosome inhibitors. Gut (in press).

\section{Call for Patients with Familial Pancreatic Disease: The EUROPAC Register}

We are establishing a national UK register (EUROPAC) of families with hereditary pancreatitis, familia pancreatic cancer and where pancreatic cancer has occurred as part of a familial cancer syndrome. This collaboration in Liverpool is between the Department of Clinical Genetics (Dr Ian Ellis) and the Academic Department of Surgery (Professor John Neoptolemos). The data and samples are collected by behalf of ESPAC (the European Study Group for Pancreatic Cancer), Professor Markus Büchler, Berne, and Professor Hans Beger, Ulm. The study will collaborate with Dr David Whitcomb of the Midwest Multicenter Pancreatitis study group in the United States. We aim to recruit families who are prepared to donate blood for DNA studies. We hope to gain a clearer understanding of the genetic relationship between hereditary pancreatitis and familial pancreatic cancer, and develop screening protocols for individuals at risk.

Hereditary pancreatitis is associated with a mutation in the recently identified cationic trypsinogen gene. This mutation renders the enzyme active within the pancreas, leading to autodigestion. Individuals with recurrent pancreatitis have a greatly increased risk of developing pancreatic cancer, and there is some evidence that DNA analysis of cells from pancreatic fluid may be valuable in detecting premalignant changes which can predict the development of pancreatic adenocarcinoma.

The criteria for inclusion in the study are as follows:

- Hereditary pancreatitis: Three relatives with chronic pancreatitis in the absence of ethanol dependence, hypercalcaemia, or an obstructive cause.

- Familial pancreatic cancer: Two first degree relatives with pancreatic adenocarcinoma. Three or more relatives with pancreatic ductal adenocarcinoma. Pancreatic ductal adenocarcinoma in any two relatives where the sum of their ages is less than 110 years.

- Other familial cancer syndromes: A single documented pancreatic ductal adenocarcinoma in any family with an established familial cancer syndrome-for example, BRCA2, FAMMM, A-T, HNPCC, or FAP.

If you know of any suitable families who may be interested in joining the study, please contact: Fiona McRonald, Clinical Genetics, Alder Hey Children's Hospital, Eaton Road, Liverpool L12 2AP. Tel: 01512525905.

Thank you for your help. 